\title{
ECRIS Sources For Highly Charged Ions
}

\author{
R. Geller
}

\author{
Département de Recherche Fondamentale, \\ Centre d'Etudes Nucléaires de Grenoble, France
}

The so-cailed Philips ionization gauge ion sources (PIGIS) were used until quite recently in heavy ion accelerators so multiply charged ions could only be obtained by incorporating a stripper to remove electrons. Electron cyclotron resonance ion sources (ECRIS) now dominate as they produce more highly charged ions.

Invented and developed mainly at Grenoble by plasma physicists, this new type of source is also robust, can be placed outside the high vacuum and does not employ filaments and arcs so it offers excellent emittance and longevity in operation [1].

Electron cyclotron resonance (ECR) ion sources have also opened up new areas of research in the low energy range, i.e. without an accelerator, by offering many kinds of highly charged ion collisions. The provision of facilities for several hundred researchers in the fields of atomic and surface science [2] has allowed the development of new instruments and methods for UV and $\mathrm{X}$-ray physics.

\section{Applications With Accelerators}

Fig. 1 shows that an immediate increase in performance is obtained by replacing a PIGIS with an ECRIS. This can be illustrated by considering the situations for cyclotrons and synchrotrons. The particle energy (in MeV per nucleon) delivered by a cyclotron is given by $W=K(q / M)^{2}$ where $q$ and $M$ denote the charge and the mass of the accelerated ion respectively, and $K$ is an efficiency factor that depends on the size of the machine and its magnetic

Professor Richard Geller has been with the French Commissariat à I'Energie Atomique (CEA), SPhAt-PSI, Bâtiment 1005, 85X, F-38041 Grenoble Cédex since 1948 and is presently a Conseiller Scientifique with interests in vacuum, plasma and fusion physics. He invented the ECR ion source in 1973 and carried out its subsequent development. He received his Doctorate from the University of Paris in 1954, has taught at Stanford University and at the University of Grenoble, and has won the Gagner Prize and the CEA Prize.

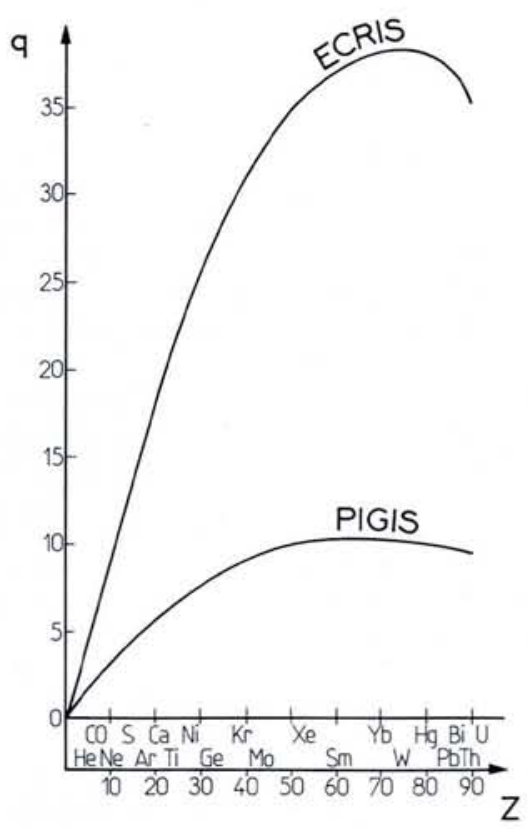

Fig. 1 - Typical values of the ion charge $q$ produced by PIG and ECR ion sources suited to accelerator applications for the elements up to atomic number $Z \approx 90$.

field strength. $K$ generally lies between 10 for smaller cyclotrons and 800 for the most powerful. By increasing the charge by a factor of 2 to 3 one can increase the particle energy by 4 to 9 times without altering anything else. This represents a bargain because the cost of such a change (i.e. replacing a PIGIS by an ECRIS) is orders of magnitude less than the cost of increasing $K$ by the same amount.

The situation is somewhat different for existing synchrotrons. Synchrotrons, like cyclotrons, can be adjusted to cope with all values of $\varepsilon=q / M$ but their injector systems (including linear accelerators) do not have the same flexibility. However, if the entire synchrotron complex is capable of accelerating $\alpha$ particles $(q / M=0.5)$, as most of them are, then they can immediately accept a variety of completely stripped ions such as ${ }^{12} \mathrm{C}^{6+},{ }^{14} \mathrm{~N}^{7+},{ }^{16} \mathrm{O}^{8+},{ }^{20} \mathrm{Ne}^{10+}$, ${ }^{32} \mathrm{~S}^{16+},{ }^{40} \mathrm{Ca}^{20+}$, etc. CERN's Super Proton Synchrotron (SPS) providing up to $200 \mathrm{GeV}$ per nucleon has therefore been able to achieve beam energies of 3.2 $\mathrm{TeV}$ with ${ }^{16} \mathrm{O}^{8+}$ ions and, more re- cently, 6.4 TeV with ${ }^{32} \mathrm{~S}^{16+}$ particles, thus permitting an investigation of quark-gluon matter to start without building a special heavy ion tevatron.

In CERN's SPS ring, an ECR ion source is coupled through a radiofrequency quadrupole to an existing linear accelerator capable of accelerating ${ }^{16} \mathrm{O}^{6+}$ and ${ }^{32} \mathrm{~S}^{12+}(\varepsilon \approx 0.37)$ to 12 $\mathrm{MeV}$ per nucleon. After passing through a foil that strips away electrons (see below), these particle are transformed into ${ }^{16} \mathrm{O}^{8+}$ and ${ }^{32} \mathrm{~S}^{16+}$ and then accelerated without further modification by the whole CERN complex comprising a booster ring, the Proton Synchrotron (PS) with $12 \mathrm{GeV}$ per nucleon and the SPS.

Using a novel type of ECR ion source and a new linac preaccelerator, an upgraded facility for $\approx 30 \mathrm{TeV}$ beams is presently being developed at CERN. Since the plan is to accelerate heavy ions up to $\mathrm{Pb}$, the new source has been dubbed the "lead injector" [3].

The impact of ECR ion sources and their capacity to open up additional fields of research in heavy ion physics is illustrated in Fig. 2 for existing accelerators. The doubly cross-hatched regions define the energies and atomic numbers that could be handled by these accelerators. The large, singly hatched regions outline the regimes that are accessible to accelerators at GSI, Darmstadt and CERN equipped with ECRIS systems.

\section{Multiply Charged Ion Production}

The only efficient technique for obtaining a significant yield of many-times ionized ions employs successive ionization. We are then led to increase $\tau$ the exposure time of the ions to a cloud of electrons of density $n$ and an impact velocity $v$. The parameter $n v \tau$ determines the charge $q$ that can be achieved. At the same time, $v$ should exceed $10^{9}$ $\mathrm{cm} \mathrm{s}^{-1}$ in order to reach the ionization potential of the inner shell electrons, i.e. several keV. Electron cyclotron resonance is ideal for this purpose as arc plasmas do not impart sufficient energy to the electrons.

Fig. 3 shows the mean charges $\langle q\rangle^{+}$for argon ions as a function of 


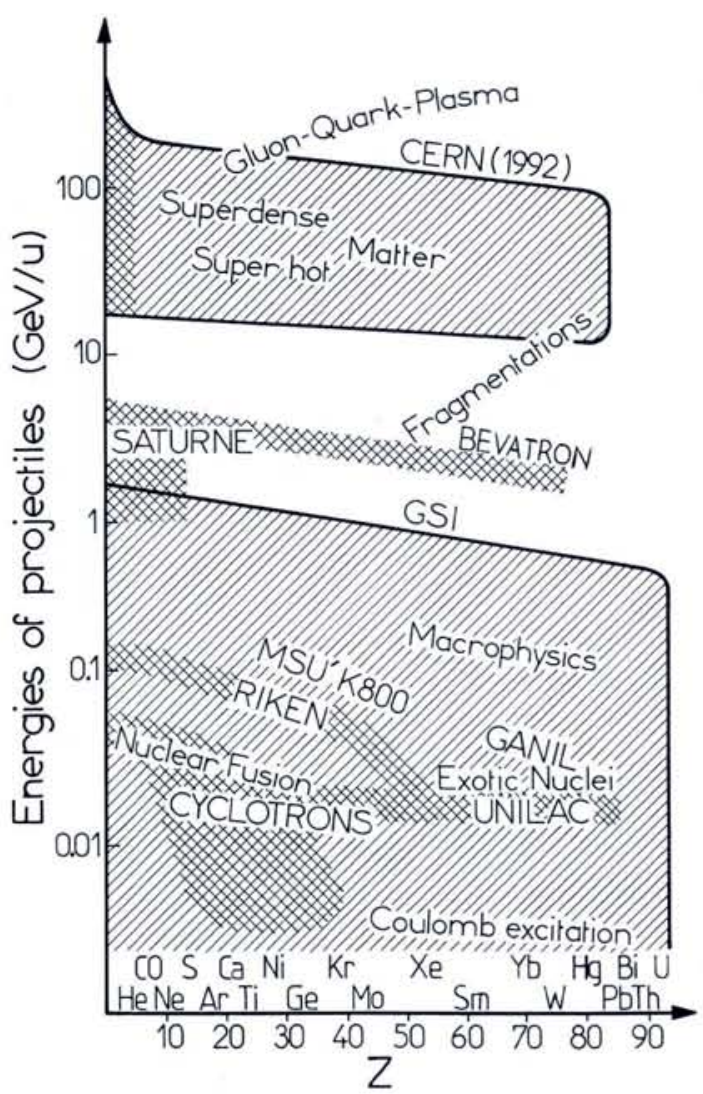

$n v \tau$. They were calculated with the help of ionization cross sections assuming that only the confinement time $\tau$ limits the achievable charge. Recombination mechanisms were not considered. It should be noted that highly charged ions arise for $n v \tau>10^{19} \mathrm{~cm}^{-2}$ and that $\langle q\rangle^{+}$increases very slowly with $n v \tau$ (on a semi-log scale) underlining the difficult of producing highly charged ions. In short, we need the highest possible electron density $n$ and confinement time $\tau$. The problem is: how can one obtain a maximum $n \tau$ value?

\section{Foilstripping}

Foilstripping is based upon the $n v \tau$ criterion but in this case one injects energetic (a few MeV per nucleon), low charge state ions through a thin foil. The foil contains in its crystalline structure atoms together with cold electrons having a density $n \approx 10^{24} \mathrm{~cm}^{-3}$. The relative interaction velocity equals the transit velocity of the accelerated ions (a few $10^{9} \mathrm{~cm} \mathrm{~s}^{-1}$ ) and the interaction time is the time the ions take to pass through the foil. Thus $\tau \approx 10^{-14} \mathrm{~s}$ with $n \tau \approx 10^{10} \mathrm{~cm}^{-3} \mathrm{~s}$ and $n v \tau>10^{19} \mathrm{~cm}^{-2}$. Two types of collisions are in competition, namely step-by-step ionization with many excited levels of the incident ion, and recombination of the multiply charged ions through electron capture. The ionization process dominates at high speeds and an ion emerging from the thin foil is highly charged.

\section{Elecron cyclotron resonance}

In ECRIS, the process is reversed. Cold plasma ions diffuse slowly through an electron cyclotron resonance plasma of hot electrons and one would obtain the same relative interaction speed as for foilstripping if ECR yielded electrons of a few $\mathrm{keV}$. This calls for an electron target plasma of $n \tau \approx 10^{13} \mathrm{~cm}^{-3} \mathrm{~s}$, similar in magnitude to that of a solid stripper. Since the electron density in ECR plasmas is usually only $10^{12} \mathrm{~cm}^{-3}$ one requires ion lifetimes of $10^{-2} \mathrm{~s}$ obtained using special magnetic confinement systems.

\section{ECR Ion Sources}

The magnetic field configuration in an ECRIS source that plays a crucial role in controlling the particle lifetime also strongly influences the RF power necessary to couple with the plasma. Improving the magnetic confinement therefore ensures more efficient ionization and production of highly charged ions. Radial magnetic mirrors in addition to axial mirrors are of great value and these multi-mirror confinement structures are usually referred to as " $B_{\min }$ structures" because they exhibit a minimum in the magnetic field at their centre. Confinement times generally exceed $10^{-3} \mathrm{~s}$ whereas $\tau$ for configurations without radial mirrors is at least 50 times smaller.

The ECR heating mechanism of the electrons can be summarized briefly as
Fig. 2 - The impact of ECRIS on heavy ion physics carried out using some well known accelerators. The doubly hatched areas are the regimes covered by the original accelerators; the singly hatched areas are those available to accelerators at CERN and GSI equipped with $E C R$ ion sources.

ARGON
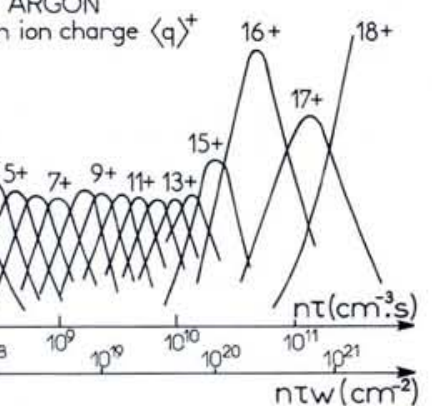
Fig. 3 - The calculated distributions of the fractions $f$ of argon ions with mean
charges $\langle q\rangle+$ as a function of $n v \tau$ for electrons of $10 \mathrm{keV}$ (velocity $v \approx 6 \times$ $\left.10^{9} \mathrm{~cm} \mathrm{~s}^{-1}\right)$. Note the very gradual increase in $\langle q\rangle+$ on the semi-logarithmic scale. Similar behaviour is calculated for all the elements.

follows: consider an empty box of undefined shape filled with microwave power (e.g. with a frequency $\omega=10$ $\mathrm{GHz}$ and wavelength $\lambda=3 \mathrm{~cm}$ ). The box can be considered as a multimode cavity if its dimensions are large with respect to the wavelength. On immersing the box in a magnetic well $\left(B_{\min }\right.$ structure) for which the magnetic field along the centreline is between $B_{\max }=$ $0.5 \mathrm{~T}$ and $B_{\min }=0.2 \mathrm{~T}$ there must exist a magnetic surface with a spherical or egg-like shape where the field strength $B_{\mathrm{ECR}}=0.36 \mathrm{~T}\left(B_{\max }\right.$ is the field on the closed magnetic surface - the walls are not intercepted - with the highest field strength and $B_{\min }$ is the minimum field at the centre of the box). This socalled ECR surface $\left(B_{\mathrm{ECR}}\right)$ gives a gyrofrequency of an electron that is $10 \mathrm{GHz}$, equal to the microwave frequency. In general, mirror ratios of $B_{\max } / B_{\min } \approx 2$ and $B_{\max } / B_{E C R}>1.5$ are required in order to confine the ECR heated plasma.

ECR necessarily occurs in a multimode cavity because there is always a component of the electric field of the waves that is perpendicular to the magnetic field lines. The electrons thereby gain mainly perpendicular momentum which adds a special ECR mirror effect, thus improving confinement.

Electrons in the magnetic well pass many times through the ECR surface where they can be either accelerated or decelerated according to their phase. They undergo global or "stochastic" heating during the many passages and rapidly reach the $\mathrm{keV}$ range (a small fraction even attains the $\mathrm{MeV}$ range).

\section{Design}

ECR ion sources generally have two stages in cascade. In the first, a cold plasma is ignited and diffuses toward the second stage bringing the hot electrons into the $B_{\text {min }}$ confinement system. The main components of a typical ECRIS with its first and second stages and the axial and radial magnetic fields 


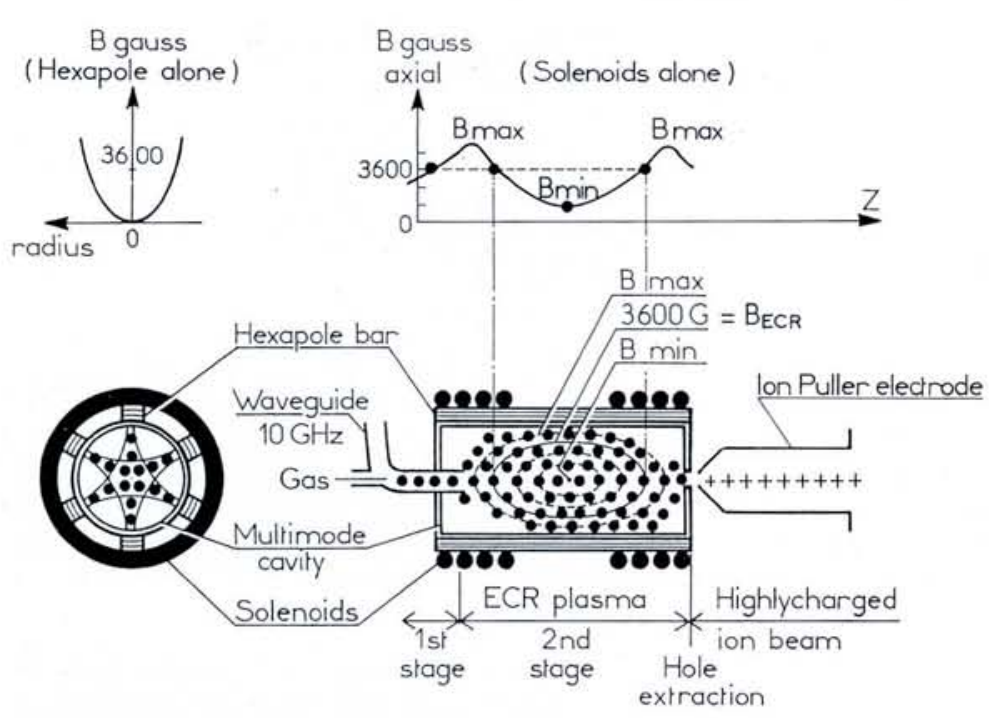

Fig. 4 - Magnetic well $\left(B_{\min }\right.$ structure) and the components for a $10 \mathrm{GHz}$ ECR ion source involving superimposition of a double axial mirror and a hexapolar radial field together with first and second stage cascading plasmas. $B_{\max }$ corresponds to the closed magnetic surface (the walls are not intercepted) with the highest field strength and $B_{E C R}$ is the "egg" shaped surface where electrons are energised. Ions are extracted from behind one of the axial magnetic mirrors.
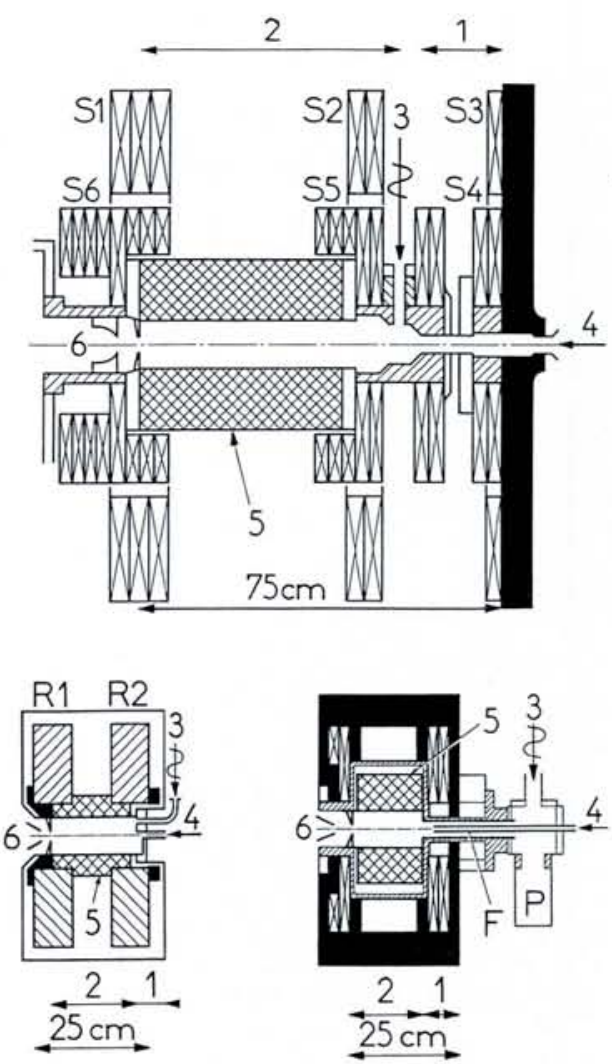

are illustrated in Fig. 4. Microwaves are injected through a $\mathrm{BeO}$ window to feed RF power into both stages. lons are extracted behind the axial magnetic mirror and the whole source, isolated to $25 \mathrm{kV}$, is kept at high voltage.

The plasma remains confined as long as the ECR surface does not intercept a solid obstacle. If not, confinement is destroyed and power is dissipated in the solid which is melted or vaporized, a feature that can be exploited for direct, in situ metal ion production. A metallic sample is placed on a movable piston for positioning at the ECR surface. (It should be noted that metallic vapour can also be obtained with ovens or by laser impact). Ion pumping due to ECR together with the effects of wall recycling in the second stage also affect the behaviour of the source. The neutral gas pressure in the second stage must be as low as possible to avoid charge exchange recombination losses.

\section{Scaling Laws:}

The Importance of $\omega$ and $B_{\max }$

Although the underlying mechanisms are not yet clear, gas mixing and wall coatings can improve the charge distribution. We have also proposed some straightforward and easily applied scaling laws that are useful for upgrading ECR ion sources [4]. Summarizing, we found the following relations, where $B=\left(B_{\max }-B_{\min }\right) / 2$ the average field strength in the trap, $\omega$ the incident RF frequency, $q_{\mathrm{opt}}$ the charge state giving

Fig. 5 - Sections through some recent types of ECR ion sources built at CEN, Grenoble. The components are: 1 - first stage plasma; 2 - second stage plasma; 3 - RF injection; 4 - gas inlet; 5 - permanent magnet solenoid; 6 - extraction electrodes; $S$-solenoid coils; $P$ - permanent magnet rings; $P$ - pumps.

Top: MINIMAFIOS which can operate at 10,14 and $16 \mathrm{GHz}$. The radial field is produced with a $\mathrm{SmCo}_{5} 0.8 \mathrm{~T}$ hexapole; the axial solenoid field is variable.

Bottom right: CAPRICE. Note the compact iron yoke and the coaxial microwave feedthrough $F$. This source operates with $B_{\max } / B_{E C R} \approx 2$ at $10 \mathrm{GHz}$. A $14 \mathrm{GHz}$ prototype is now available.

Bottom left: NEOMAFIOS. Note the absence of coils and pumps. The magnetic structure is made entirely of $\mathrm{NdFeB}$ so this $8-10 \mathrm{GHz}$ source consumes only $\approx 5 \mathrm{~kW}$.

the maximum ion current, $1^{\mathrm{q}}$ the value of this current and $M$ the mass of the ion:

$$
\begin{gathered}
n v \tau \sim B^{1.5} ; q_{\mathrm{opt}} \sim \log B^{1.5} \\
\quad \text { for variable } B ; \\
n v \tau \sim \omega^{3.5} ; q_{\mathrm{opt}} \sim \log \omega^{3.5}
\end{gathered}
$$

for variable $\omega$ and $B$;

$$
l^{\mathrm{q}} \sim \omega^{2} M^{-1} \text {. }
$$

These relations indicate that improvements in performance are possible by increasing $\omega$ and $B_{\max }$ and this has been confirmed in experiments where the $B_{\max } / B_{\mathrm{ECR}}$ ratio was increased and by varying $\omega$ from 6.4 to $18.0 \mathrm{GHz}$. The scaling laws, although approximate, seem to be relevant to a certain extent and they allow extrapolation, for instance to $30 \mathrm{GHz}$. Such an extrapolation is the basis of a joint project between CEN, Grenoble and Michigan State University, USA involving the application of advanced technology - superconducting coils for $B$ and gyrotrons for raising the frequency $\omega$ [5].

\section{ECRIS Prototypes}

More than 30 ECR ion sources are in operation around the world. The majority of them were built in small batches at CEN, Grenoble. All existing ECR ion sources (including those not built at Grenoble) are imitations, some successful and others less so, of the following Grenoble prototypes: I) SUPERMAFIOS, II) CRYOMAFIOS, III) MINIMAFIOS, IV) CAPRICE, V) NEOMAFIOS [1,6,7]. Each of these variants is based upon the ECR principle described above. They have various sizes and work at different frequencies depending on the users' needs and resources. However, their somewhat different performance characteristics can always be described in terms of the scaling laws.

Variations between the sources arise mainly from the types of materials used to construct the $B_{\min }$ structure comprising solenoids $\mathrm{S}$ and hexapoles $\mathrm{H}$ (made of copper wire c, superconductors sc, permanent magnets $p$ and iron yokes i). 

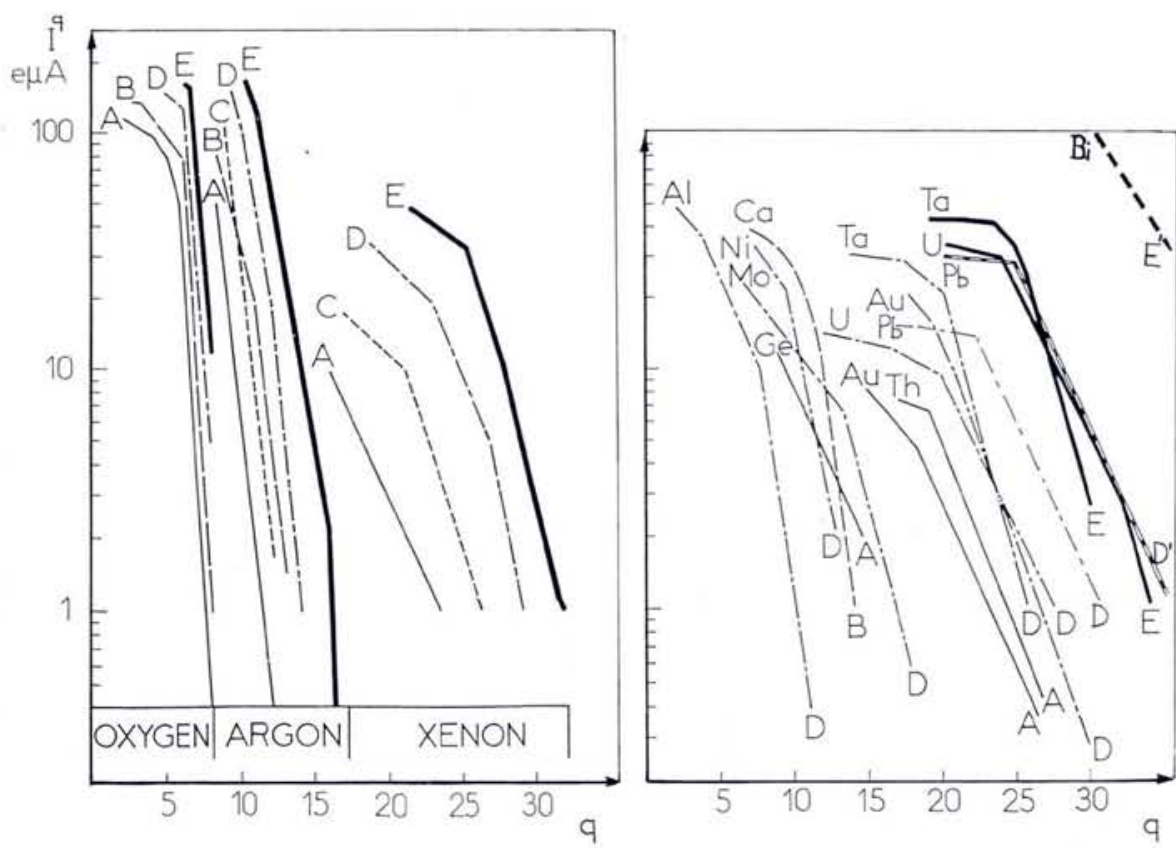

Fig. 6 - Typical values of the ion currents la obtained with various ECR ion sources as a function of the charge states $q$ for different elements. ECRIS types are: A - NEOMAFIOS $(8 \mathrm{GHz}) ; B$ - MINIMAFIOS (10 GHz); C - CAPRICE (10 GHz, normal B mode); D - CAPRICE $\left(10 \mathrm{GHz}, B_{\max } / B_{E C R}>2\right)$; $D^{\prime}$ - CAPRICE (14 GHz, $\left.B_{\max } / B_{E C R}>2\right) ; E-M I N I M A F I O S(16 \mathrm{GHz})$; E - MINIMAFIOS (18 GHz); $E^{\prime}$ - MINIMAFIOS (18 GHz, short pulses).

Left: Gaseous elements. Right: Solid state elements.

The prototypes, illustrated in Fig. 5, can be designated as follows:

$$
\begin{aligned}
& \text { I) } \mathrm{S} . c+H . c \text {, } \\
& \text { II) } \mathrm{S} . \mathrm{sc}+\mathrm{H} . \mathrm{sc} \text {, } \\
& \text { III) } \mathrm{S.c}+\text { H.p, } \\
& \text { IV) S.c + H.p + i, } \\
& \text { V) S.p + H.p. }
\end{aligned}
$$

Group IV) is presently very popular because it allows compact sources with a smaller power consumption than III). Type II) is the most promising in spite of its larger size and higher cost because it can reach the highest values of $\omega$ and $B_{\max }$ whereas $\mathrm{V}$ ) is limited to $0.5 \mathrm{~T}$ by the properties of currently available rare-earth magnets. However, Type $\mathrm{V}$ gives a very respectable performance with a total power of $\approx 5 \mathrm{~kW}$ thus facilitating installation, even on high voltage platforms. Fig. 6 summarizes the performance of some of the prototypes.

\section{Pulsed ECRIS for Synchrotrons}

Many forms of beam shapes can be realized by equipping ECRIS for continuous wave (CW) operation with external beam chopping. However, pulsing the RF power offers superior opportunities and the best results are obtained if the duration of the RF pulse $\tau_{\mathrm{RF}}$ is long $\left(\tau_{\mathrm{RF}}>10 \mathrm{~ms}\right)$ and the duty factor is kept below $30 \%$. This improvement is due to a reduced amount of thermally activated release of gas from the walls

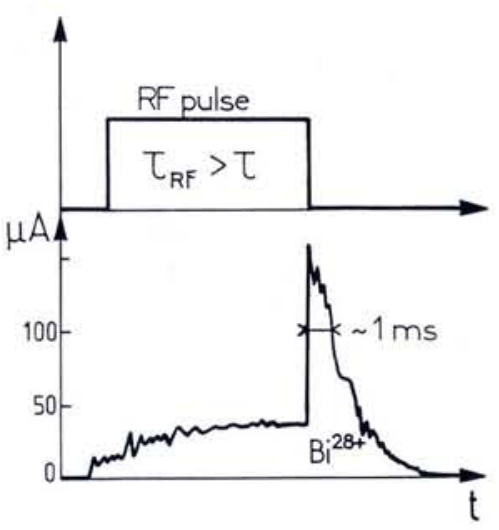

Fig. 7 - Short ion pulses for synchrotron injection: the current of $\mathrm{Bi}^{28+}$ ions is plotted as a function of time. The axial mirror effect is cancelled when the RF pulse ends, thereby releasing a burst of additional ions from the bulk of the plasma (MINIMAFIOS, $18 \mathrm{GHz}$ ).

of the device leading to weaker charge exchange recombinations. However, the Grenoble team predicted and demonstrated [6] that there exists a short, intense ion burst at the end of each RF pulse (Fig. 7). This burst is made up ions that are released axially from the bulk of the plasma and are no longer contained by the ECR mirror effect when the RF pulse terminates.

By optimizing the tuning one can obtain peak intensities up to 10 times larger than $\mathrm{CW}$ intensities. This is very important because the short pulses are suitable for synchrotron injection. CERN's "lead injector" will exploit the discovery. Pulsing the feed gas, recycling solid material from the walls and electrostatic "pushing" yield further improvements [8].

\section{Ultra-relativistic Electrons For Atomic Physics}

Stochastic ECR yields - in addition to the bulk of electrons with energies in the $\mathrm{keV}$ range - roughly $1 \%$ of highly relativistic electrons which are long living because they are nearly collisionless (the cross sections for step-by-step ionization being very small). These hot electrons are capable of ejecting electrons from the K-shell of a target in a single impact. The study of electronic rearrangements inside this target through recombination, radiation, Auger cascades, etc. offers an original approach for understanding these processes. Therefore, not only the ion beam issuing from an ECR ion source but also the hot radiation produced by the hot, relatively dense ECR plasma are useful in atomic physics.

\section{Conclusions}

ECR ion sources have opened up new fields of research in particle science. They now represent the dominant ion sources for heavy ion accelerators and ECRIS beams are today injected into many types of accelerators (RQF, linear accelerators, cyclotrons, tandems and synchrotrons).

They play an important rôle in reducing the cost of facilities for heavy ion physics by enabling considerable reductions in the size of new accelerators and of upgrades of existing machines to significantly higher energies. Their outstanding properties include the production of sufficiently intense beams of highly charged ions, with good stability and longevity, for accelerators used in high energy physics and for experiments in atomic physics.

\section{REFERENCES}

[1] Geller R., Nucl. Sci. NS 23 (1976) 904; NS 26 (1979) 2120.

[2] Proc. Int. Conf. on Physics of Multiply Charged lons and Int. Workshop on ECRIS: Ed. S. Bliman, J. de Physique 50, Colloque Cl (1989).

[3] Haseroth H., Particle World 1 (1989) 14.

[4] Geller R., Ref. (2), p. 887.

[5] Antaya T., Ref. (2), p. 707

[6] Melin G. et al., Ref. (2).

[7] Jacquot B. et al., Nucl. Inst. and Meth. A269 (1988) 1.

[8] Briand P. et al., Nucl. Inst. and Meth. (to be published). 